same cartilage lesions in order to establish the validity of this procedure.

Methods Articular cartilage of medial compartments of three OA knee joints were magnified evaluated and recorded using a 2,7 mm scope. 34 different areas of lesion were individualised and fissures; III: deep fissures; IV: exposure of subchondral bone.73 sections ofarthroscopically normal and OA cartilage were microscopically examined and graded twice by two observers according to Mankin's histopathological grading system (014)(MHHGS). Mathematic categorization in five groups was done to correlate with chondroscopic grades of lesion: normal (0-2), mild (3-5), moderate (6-8), severe (9-11), and very severe (12-14).

Results Intra and interobserver variability for MHHGS score was determined with Sperman's correlation coefficient for both observers: $\mathrm{r}=0,890 \mathrm{p}<0,001 ; \mathrm{r}=0,835 \mathrm{p}<0,001$ and $\mathrm{r}=$ $0,906 \mathrm{p}<0,001 ; \mathrm{r}=0,822 \mathrm{p}<0,001$. Average MHHGS score for chondroscopic grade $0(1,7 \pm 0,59 \mathrm{n}=20)$, grade $\mathrm{I}(3,62 \pm$ $2,33 \mathrm{n}=28)$, grade II $(6,82 \pm 1,75 \mathrm{n}=52)$ were as expected, but for grade III $(8,13 \pm 2,13 \mathrm{n}=34)$ and grade IV $(11,5 \pm$ $3,18 \mathrm{n}=14)$ were lower. Correlation between CHGS and MHHGS scores were measure computing the kappa index for categorical variables for both observers: $\mathrm{kp}=0,50 \mathrm{kp}=0,58$.

Conclusion CHGS score of cartilage might be considered a relevant tool of OA outcome for research. The low tendency to overstimate lesions in advanced stages may be related to the mathematic categorization of the histopathological score.

\section{SAT0080 INSIGHT INTO THE STRUCTURAL BASIS OF SELECTIVE CYCLOOXYGENASE-2 INHIBITION}

${ }^{1} \mathrm{G}$ Trummlitz, ${ }^{2} \mathrm{H}$ Wittneben. ${ }^{1}$ International Project Management; ${ }^{2}$ Chemical Research, Boehringer Ingelheim, Biberach, Germany

\subsection{6/annrheumdis-2001.455}

Background Cyclooxygenase-1 and -2 (COX-1/COX-2) catalyse the rate-limiting step in prostaglandin (PG) synthesis and are the targets of non-steroidal anti-inflammatory drugs (NSAIDs). The evidence is strong that the selective COX-2 inhibitors have reduced GI side effects in patients treated for rheumatic conditions, including osteoarthritis.

Objectives The molecular basis of binding affinities and COX-2/ COX-1 selectivity are explored using an approach that combines experimental results from X-ray analyses and docking experiments from computational chemistry.

Methods The reported X-ray structures of COX-1 and COX-2 were used for computational optimisation of the interaction between the different NSAIDs and COX isoforms using the established computer program Sybyl/Molcad.

Results The inhibition of COX can occur by compounds with high degree of structural diversity and various types of mechanisms. The results show that COX-2 selective inhibitors are belonging to several different, structural distinct classes: arylalkanoic acids, enolcarboxamids, acidic sulfonamids and diarylheterocycles.

Two fundamental differences between the active site of COX2 and COX-1 are responsible for the COX-2 selectivity and are caused by several amino acid substitution in COX-2 vs. COX-1: The volume of the COX-2 channel is extended by the substitution of Iso 523 by Val 523, Iso 434 by Val 434, His 513 by Arg 513 and Phe 503 by Leu 503.
COX-selective diaryl-heterocycles (DuP 697, celecoxib, rofecoxib) are exploiting the side pocket and enolcarboxamid (meloxicam) and acidic sulfonamids (L745,733, NS 398, nimesulide) the extra space at the top of the channel.

Conclusion This work illustrates the value of novel docking procedure for determining the binding of NSAIDs to the COXenzyme. The structural insights into the binding mechanism indicate that COX-2 selectivity can be obtained by at least three chemically distinct classes of NSAIDs of which meloxicam, nimesulide and celecoxib are representatives.

\section{SAT0081 EFFECT OF BODY MASS INDEX AND BONE MINERAL DENSITY ON KNEE OSTEOARTHRITIS}

A Bilgici, O Kuru, G Alayli, N Yildiz. Physical Medicine and Rehabilitation, Ondokuz Mayis University Medical Faculty, Samsun, Turkey

10.1136/annrheumdis-2001.456

\section{Background}

Objectives The aim of this study was to investigate the influence of the bone mineral density (BMD) and body mass index (BMI) on the knee osteoarthritis (OA).

Methods Seventyfive patients were taken in this study. BMD was measured using dual X-ray absorptiometry (Dexa). Measurements of the spine were made at L1-L4 and of the hip at three sites (femoral neck, Ward's region and trochanter). The BMI, an indicator of obesity was calculated as the weight divided by the height squared $\left(\mathrm{kg} / \mathrm{m}^{2}\right)$. The knee $\mathrm{OA}$ was assessed from a weight-bearing anteroposterior radiograph. Osteophytes and joint space narrowing were evaluated seperately. Individual radiographic features (IRF) rating method was used for grading of osteophytes (0-3) and joint space narrowing (0-3).

Results The mean age of the women was 67 years (48-75) and of the 23 men was 61 years (45-66). There was a highly positive correlation between BMD and OA with $\mathrm{BMI}(\mathrm{p}<0.01, \mathrm{p}<$ 0.01 respectively). The density of the lumbar spine and the 3 proximal femur sites was significantly larger in men than in women. Mean femoral BMD was correlated with joint space narrowing but not with osteophytosis $(\mathrm{p}<0.05)$. There was no relationship between the density of the lumbar spine with knee OA.

Conclusion There is a clear link between BMD and BMI with the knee OA. These results suggest that mechanical factors are important in the pathophysiology of these diseases.

\section{SAT0082 A RANDOMISED, DOUBLE-BLIND, PLACEBO- CONTROLLED, ORAL SINGLE DOSE, DOSE ESCALATION TRIAL, INVESTIGATING THE SAFETY, TOLERABILITY AND PHARMACOKINETICS OF SVT-2016 IN HEALTHY MALE SUBJECTS. PRELIMINARY REPORT}

${ }^{1} \mathrm{R}$ López Bertrán, ${ }^{1} \mathrm{M}$ Aliaño, ${ }^{2} \mathrm{C}$ Lagunas, ${ }^{3} \mathrm{~S}$ Febbraro, ${ }^{3} \mathrm{EA}$ Ferguson. ${ }^{1} \mathrm{Medical}$ Department; ${ }^{2}$ Preclinical Research, Laboratorios SALVAT, S.A., Esplugues de Llobregat, Spain; ${ }^{3}$ SIMBEC Research Limited, Merthyr Tydfil Mid Glamorgan, UK

\subsection{6/annrheumdis-2001.457}

Background As a pivotal enzyme in the inflammatory response, inhibition of cyclooxygenase is an established mechanism of action for many anti-inflammatory agents. Non-selective inhibition of COX reduces pain, fever and inflammation but is often associated with gastrointestinal or renal injury. In the past decade, the existence of multiple isoforms of COX has become 